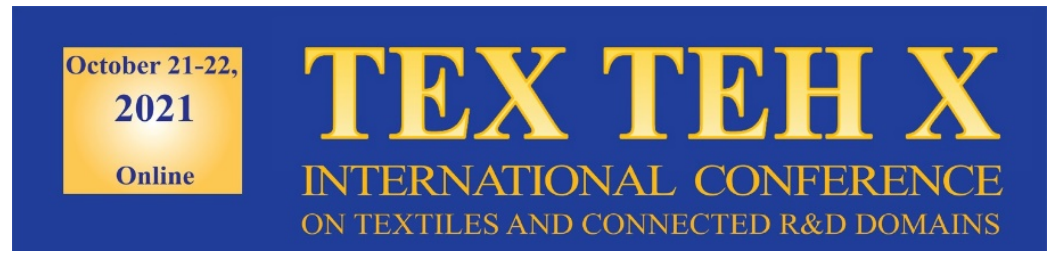

\title{
AQUEOUS SOLUTIONS OF CHITOSAN: VISCOMETRIC AND FLOCCULATION PROPERTIES
}

\author{
DOI: 10.35530/TT.2021.21
}

\section{C.-E. Brunchi*, L. Ghimici}

\author{
Natural Polymers, Biocompatible and Bioactive Materials, "Petru Poni” Institute of Macromolecular \\ Chemistry, Romania \\ (E-mail: brunchic@icmpp.ro, lghimici@icmpp.ro)
}

\begin{abstract}
The hydrodynamic and flocculation properties of aqueous solution of chitosan (CS) were investigated at room temperature. The viscometric data of dilute CS solutions were discussed in terms of Wolf method as a function of polymer concentration, salt nature ( $\mathrm{NaCl}, \mathrm{NaNO}_{3}$, and $\left.\mathrm{CaCl}_{2}\right)$ and concentration. The flocculation properties in emulsions of some commercial pyrethroid insecticides (Fastac 10 EC (F), Decis (Dc) and Karate Zeon (KZ)) were evaluated. The viscosity measurements reveal that the experimental data fit well with the Wolf model and the [ $\eta]$ values decrease in salt aqueous solutions. For the same insecticide concentration (0.02\%, $v / v$ ), UV-Vis spectroscopy measurements show maximum removal efficiency around $90 \%$ for Fastac 10EC and Decis and $80 \%$ for Karate Zeon. The residual KZ absorbance decreased with the increase of insecticide concentration in the initial emulsion, from $80 \%$ for the lowest concentration $(0.02 \%, v / v)$ to around $90 \%$ for the highest one $(0.06 \%, v / v)$. The supernatant zeta potential dependence on the chitosan dose pleads for the charge neutralization as the main mechanism for the flocculation of pyrethroid insecticide particles.
\end{abstract}

Keywords: chitosan, flocculation, insecticides, salt solution, viscosity

\section{INTRODUCTION}

Chitosan is a well-known cationic polysaccharide made of glucosamine and a fraction of acetylglucosamine residues [1]. The main characteristics of chitosan, namely biodegradability, nontoxicity, hydrophilicity, easy chemical derivatisation, etc., are the consequence of the $-\mathrm{OH}$ and primary amino groups presence in its chemical structure. By virtue of these properties, chitosan has found applications in many fields such as medical, agriculture, food, textile, cosmetics, pharmaceutical, papermaking industries [2-4]. Its using in the above mentioned fields has challenged the researchers to investigate the dynamic (viscosity, rheology, etc.) and electrochemical (conductometry, activity, etc.) properties in solution with different characteristics $(\mathrm{pH}$, ionic strength, etc.). The viscosity measurements provide valuable information about interactions between polymer chains or with solvent molecules, conformational transition of the chains and some hydrodynamic parameters of the polymer chains in solution [5,6]. In this context, a first aim of this paper was the investigation of some salts effect on the hydrodynamic properties of CS chains in aqueous solution and determination of the intrinsic viscosity by the Wolf method [7]. Also, the high ability of CS to interact with a wide range of compounds (salts, clays, oils, dyes, metal ions, etc.) has directed its application in the wastewater treatment field [2,8]. The presence of pesticides, a group of highly toxic materials for the living organisms in the environment (surface water and industrial/agriculture wastewater), has determined an increasing interest in their removal by 
different physico-chemical methods [9,10]. However, the application of soluble polysaccharides including chitosan in removal of pesticides from wastewater was less investigated [9, 10]. The good removal efficiency (around 90\%) obtained when dextran [9] and pullulan [10] derivatives were used as flocculants encouraged us to test the efficiency of chitosan solution in decreasing of some commercial pyrethroid insecticides content from wastewater. The insecticides chosen in this work (widespread applied in agricultural crops, forestry as well as in public and animal health) were Fastac 10 EC, Decis and Karate Zeon. The flocculation efficacy of CS in pyrethroid insecticides emulsions was followed by UV-Vis spectroscopy as a function of dosage (polymer dose represents the flocculant concentration in its mixture with pesticide emulsions). In addition zeta potential measurements were performed in order to establish the possible mechanisms that control the insecticides removal process.

\section{MATERIALS AND METHODS}

\subsection{Materials}

Chitosan (CS, chemical structure in figure 1, a, acetic acid (AcOH) and used salts $\left(\mathrm{NaCl}, \mathrm{NaNO}_{3}\right.$ and $\mathrm{CaCl}_{2}$ ) were purchased from Sigma-Aldrich, and were used as received.

The viscometric average molar mass of CS $\left(M_{v}=189 \mathrm{kDa}\right)$ was estimated from the intrinsic viscosity, $[\eta]$, according to the equation proposed by Gamzazade et al. [11]:

$$
[\eta]=1.38 \times 10^{-4} M_{v}^{0.85}\left(\mathrm{dL} \cdot \mathrm{g}^{-1}\right)
$$

Degree of acetylation (DA) of chitosan was evaluated by infrared spectroscopy (Vertex 70 Bruker FTIR spectrometer). Transmission spectra were recorded in KBr pellets. For DA determination, Equation (2) was used taking the $1420 \mathrm{~cm}^{-1}$ band as reference and the band located at $1320 \mathrm{~cm}^{-1}$ as characteristic band for the $\mathrm{N}$-acetylglucosamine [12]:

$$
\mathrm{A} 1320 / \mathrm{A} 1420=0.3822+0.03133 \mathrm{DA}
$$

An average value of $\mathrm{DA}=15 \%$, obtained from three measurements, was taken into consideration.

The insecticide called in the paper $F$ is commercially available as Fastac 10 EC (BASF), in small bottles: $2 \mathrm{ml}$ solution ( $\alpha$-Cypermethrin: $100 \mathrm{~g} \cdot \mathrm{l}^{-1}$; solvent naphtha (petroleum), light arom.) (chemical structure in figure 1, b). The insecticide called in the paper $D c$ is commercially available as Decis, in vials with $2 \mathrm{~mL}$ solution (Deltamethrin: 50 $\mathrm{g} \cdot \mathrm{L}^{-1}$; solvent naphtha (petroleum), heavy arom.) (chemical structure in figure $1, \mathrm{c}$ ). The insecticide called in the paper $K Z$ is commercially available as Karate Zeon (Syngenta Limited, England) in small bottles/vials: $2 \mathrm{ml}$ solution (lambda-Cyhalothrin: $50 \mathrm{~g} \cdot \mathrm{l}^{-1}$; solvent naphtha (petroleum), heavy aromatics, propylene glycol) (chemical structure in figure 1, d).

\subsection{Methods}

\section{Viscometric measurements}

An automatic viscometer (Instrument LAUDA LMV 830) equipped with a water bath (ECO ET 155) and an Ubbelohde suspended-level viscometer with capillary diameter of $0.64 \mathrm{~mm}$ (Type 531 10, Schott-Geräte) was used to perform the viscometric measurements for the aqueous solutions of CS (in the absence/presence of salts). The measurements were made at room temperature $\left( \pm 0.01^{\circ} \mathrm{C}\right)$ and repeated at least twice in order to check the reliability of the data, which was $\pm 3 \%$. The chitosan solutions in aqueous $\mathrm{AcOH}(1 \%, \mathrm{v} / \mathrm{v})$ and salt 
solution with different concentration $(0.1,1$ and $10 \mathrm{mM})$ were prepared one day before the viscometric measurements.

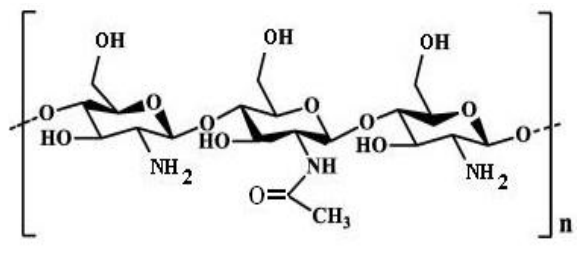

$\boldsymbol{a}$

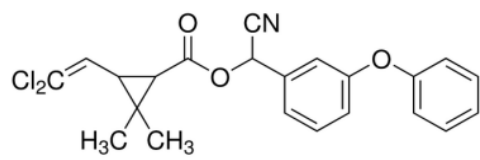

b

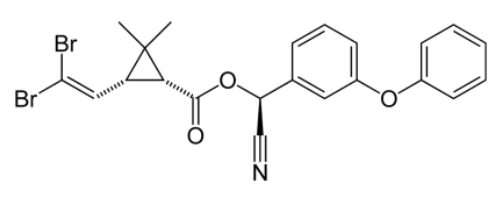

$C$

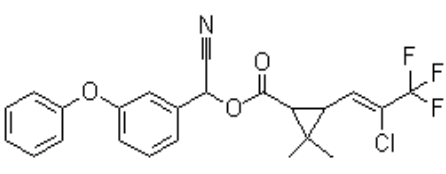

$d$

Figure 1. Chemical structure of: a - chitosan and used insecticides b - alpha-Cypermethrin; c - Delthametrin; d - lambda-Cyhalothrin

\section{Flocculation procedure}

The stock chitosan solution was prepared (CS powder was dissolved in aqueous solution of acetic acid $(1 \%, \mathrm{v} / \mathrm{v}))$ under magnetic stirring for $24 \mathrm{~h}$ at room temperature, 1 day before the flocculation experiments. Insecticide emulsions were also obtained in highly purified deionized water with the next concentrations $\left(c_{\mathrm{ie}} \%, \mathrm{v} / \mathrm{v}\right)$ : (i) $F$ emulsion (the initial $\mathrm{pH}=4.4, \zeta=-24.6 \mathrm{mV}$ and $c_{\mathrm{ie}}=0.02$ ); (ii) Dc (the initial $\mathrm{pH}=4.5, \zeta=-28.2 \mathrm{mV}$ and $c_{\mathrm{ie}}=$ 0.02); (iii) $\mathrm{KZ}$ (the initial $\mathrm{pH}=4.5, \zeta=-26.5 \mathrm{mV}$ and $c_{\mathrm{ie}}=0.02,0.04$ and 0.06). The concentration of polymer in its stock solution was $1 \mathrm{~g} \cdot \mathrm{L}^{-1}$. In order to obtain stable insecticides emulsions, the stock ones (sample volume of $500 \mathrm{~mL}$ ) were sonicated for 15 min using an ultrasonicator with VCX 750 SONICS, USA. The flocculation measurements were carried out at room temperature, using a Cole Parmer stirrer/hotplate 9 places, according to the method already described [9]. After $1200 \mathrm{~min}$ settling time, $10 \mathrm{ml}$ of supernatant was taken out for both the absorbance (spectrophotometer SPECOL 1300 Analytik Jena) and zeta potential measurements (Zetasizer Nano-ZS, ZEN-3500 model (Malvern Instruments, Malvern, England). The absorbance measurements were recorded at wavelength $(\lambda, \mathrm{nm})=$ $276 \mathrm{~nm}$ for F, $267 \mathrm{~nm}$ for Dc and $270 \mathrm{~nm}$ for KZ. The pesticide removal was expressed as percent of the initial absorbance recorded for the insecticide particle emulsions, at time zero (without polymer). Triplicate experiments were made and the mean values were calculated. Standard deviation determined for the experiments was $\pm 4 \%$.

\section{RESULTS AND DISCUSSION}

\subsection{Viscosity data}

The viscometric behaviour of aqueous solution of CS as a function of polymer concentration in terms of reduced viscosity $\left(\eta_{\mathrm{sp}} / c\right)$ and relative viscosity $\left(\ln \eta_{\mathrm{r}}\right)$ is presented in figure 2 .

$\eta_{\mathrm{sp}}$ is the specific viscosity $\left(\eta_{s p}=\eta_{r}-1\right)$ which shows the change in viscosity of the polymer solution by the addition of the solvent. $\eta_{r}$ represents the relative viscosity determined as the ratio between by the viscosities of the polymer solution and the solvent (experimentally determined as $t / t o$, where $t$ and $t_{0}$ represents the flow time of polymer solution and solvent, respectively). 
The plot of $\eta_{\mathrm{sp}} / c-c$ (figure 2, a) points out the following aspects: $i$ ) in salt solutions, the values of reduced viscosity decrease linearly with CS concentration like the neutral polymers; ii) in the absence of salt, the reduced viscosity increases at high dilution (namely, for polymer concentrations lower than $0.002 \mathrm{~g} \cdot \mathrm{dl}^{-1}$ ) that is characteristic for the polyelectrolyte behaviour. This is due to the electrostatic repulsion interactions between the charged groups $\left(-\mathrm{NH}^{+}\right)$which lead to the chain expansion; consequently, the determination of the intrinsic viscosity ([ $\eta])$ by the Huggins method becomes difficult. For this reason we have chosen to determine the $[\eta]$ according to the method proposed by Wolf [7].
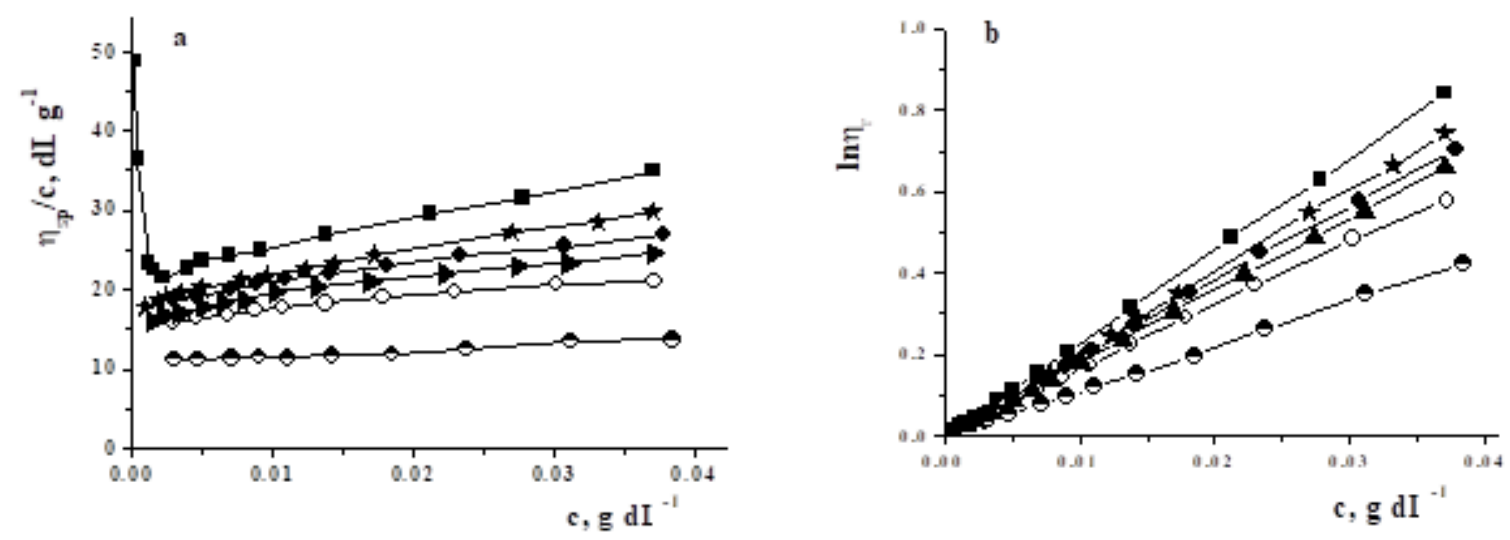

Figure 2. Dependence on: a - chitosan concentration, $c$, of reduced viscosity and b - relative viscosity in $\mathrm{AcOH}$ (full square) and in aqueous salt solutions: $0.1 \mathrm{mM} \mathrm{NaCl}$ (full circle), $1 \mathrm{mM} \mathrm{NaCl}$ (emty circle), $10 \mathrm{mM} \mathrm{NaCl}$ (half empty circle), $0.1 \mathrm{mM} \mathrm{NaNO}_{3}$ (full star) and $0.1 \mathrm{mM} \mathrm{CaCl} 2$ (full triangle).

This model allows the evaluation of the $[\eta]$ values from the initial slope of dependence of $\ln \eta_{r}$ as a function of polymer solution concentration (c) at sufficiently low shear rates and $c$, according to the following equation:

$$
\ln \eta_{\mathrm{r}}=\frac{c[\eta]+B c^{2}[\eta][\eta]^{*}}{1+B c[\eta]}
$$

where: $\eta_{\mathrm{r}}=$ the relative viscosity, $[\eta]=$ the intrinsic viscosity, $B=$ the viscometric interaction parameter and $[\eta]^{*}=$ the characteristic specific hydrodynamic volume which incorporate the effects of electrostatic interaction.

Figure 2, b presents the evaluation of the experimental data from figure 2, a according to the Wolf model. The results demonstrate that all of calculated lines coincided well with the experimental points proving the ability of Equation (3) to determine the intrinsic viscosity and to describe the viscosity behaviour of CS in the absence/presence of salts.

Table 1 emphasizes (i) the higher values of $[\eta]$ for chitosan in AcAc solution than those in all salt solutions (the same concentration); (ii) for the same salt $(\mathrm{NaCl})$, the decrease of $[\eta]$ with enhancing its concentration.

By adding salt, the electrostatic repulsion interactions between charged groups are screened, chains adopt a more compact conformation and thus intrinsic viscosity values decrease. The macromolecular chain conformation in solution is reflected in the value of $c^{*}$. In the present study, the increase of $c^{*}$ from 0.04 to $0.093 \mathrm{~g} \cdot \mathrm{dL}^{-1}$ with salt concentration reveals the transition of chitosan chains from the extended conformation to a coiled one (table 1). 
Table 1. Viscometric parameters determined by eq. 1 for CS aqueous solutions

\begin{tabular}{|c|c|c|}
\hline Solvent/salt concentration & $\begin{array}{c}{[\boldsymbol{\eta}]} \\
\left(\mathbf{d l} \cdot \mathbf{g}^{\mathbf{1}} \mathbf{)}\right.\end{array}$ & $\begin{array}{c}\boldsymbol{c}^{*}=[\boldsymbol{\eta}]^{-\mathbf{1}} \\
\left(\mathbf{g} \cdot \mathbf{d l}^{-1} \mathbf{)}\right.\end{array}$ \\
\hline $\mathrm{AcOH} 1 \%$ & 22.7 & 0.044 \\
$\mathrm{AcOH}(1 \%) / \mathrm{NaCl}(0.1 \mathrm{mM})$ & 19.534 & 0.050 \\
$\mathrm{AcOH}(1 \%) / \mathrm{NaCl}(1 \mathrm{mM})$ & 16.718 & 0.059 \\
$\mathrm{AcOH}(1 \%) / \mathrm{NaCl}(10 \mathrm{mM})$ & 10.740 & 0.093 \\
$\mathrm{AcOH}(1 \%) / \mathrm{NaNO}_{3}(0.1 \mathrm{mM})$ & 19.954 & 0.051 \\
$\mathrm{AcOH}(1 \%) / \mathrm{CaCl}_{2}(0.1 \mathrm{mM})$ & 17.793 & 0.056 \\
\hline
\end{tabular}

\subsection{Flocculation data}

Influence of chitosan dose

One of the most important parameters which gives an indication of the performance in coagulation/flocculation process of a compound (inorganic salts or polymer) is the optimum dose (dose $e_{o p}$ ), which is the polymer dose corresponding to the maximum removal efficiency. Hence, the percent of insecticides removal dependence on the chitosan dose was followed for all of the insecticides under study; insecticide emulsions with $c_{\mathrm{ie}}(\%, \mathrm{v} / \mathrm{v})=0.02$ were used (figure 3, a).

The maximum removal efficiency (RE\%) more than $90 \%$ has been found in the polymer dose interval between $1.2 \mathrm{mg} \cdot \mathrm{l}^{-1}$ and $1.4 \mathrm{mg} \cdot \mathrm{l}^{-1}$ for $F$ and between $1 \mathrm{mg} \cdot \mathrm{l}^{-1}$ and $1.1 \mathrm{mg} \cdot \mathrm{l}^{-1}$ for $D c$. A slightly lower efficacy of chitosan was found in case of $K Z$, around $80 \%$ at a polymer dose of $0.8 \mathrm{mg} \cdot \mathrm{l}^{-1}$. The explanation for this behavior could be found looking at the chemical structure of the partners implied in the systems investigated. Thus, the chitosan sample used in this investigation is a high charge density polycation (DA $=15 \%$, see Materials and Methods part, 2.1. Materials subsection); the pKa of chitosan with a degree of deacetylation above $70 \%$ is around 6.3-6.4 [14].
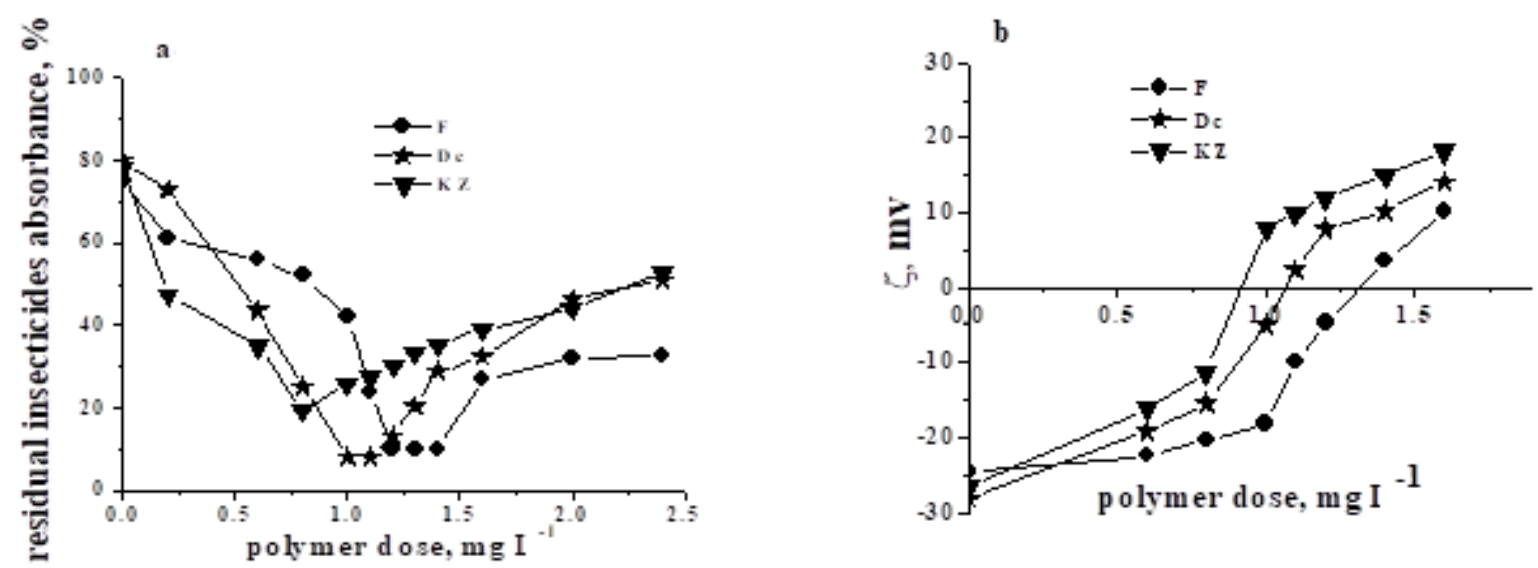

Figure 3. Graph of: a - residual insecticides absorbance (\%); b - zeta potential ( $\zeta$ ) dependence on the chitosan dose; $c_{\text {ie }}(\%, \mathrm{v} / \mathrm{v})=0.02$

On the other hand, the insecticides ( $i$ ) $\alpha$-Cypermethrin, the active ingredient of $F$, is a racemic mixture of (S)-a-cyano-3-phenoxybenzyl-(1R,3R)-3-(2,2-dichlorovinyl)-2,2dimethylcyclopropanecarboxylate and (1R)-a-cyano-3-phenoxybenzyl-(1S,3S)-3-(2,2dichlorovinyl)-2,2-dimethylcyclopropanecarboxylate, (ii) Deltamethrin- the active ingredient of $D c$ is [(S)-Cyano-(3-phenoxyphenyl)-methyl] (1R,3R)-3-(2,2-dibromoethenyl)-2,2dimethyl-cyclopropane-1-carboxylate and (iii) lambda-Cyhalothrin- the active ingredient of $\mathrm{KZ}$, is a mixture of (1R)-lambda-cyano-3-phenoxybenzyl (1S)-cis-3-[(Z)-2-chloro-3,3,3trifluoropropenyl]-2,2-dimethylcyclopropanecarboxylate and (S)-lambda-cyano-3phenoxybenzyl (1R)-cis-3-[(Z)-2-chloro-3,3,3-trifluoropropenyl]-2,2 dimethylcyclopropane- 
carboxylate; zeta potential values of the insecticide particles emulsions without chitosan are $\zeta$ $=-24.6 \mathrm{mV}$ for $F, \quad \zeta=-28.2 \mathrm{mV}$ for $D c$ and $\zeta=-26.5 \mathrm{mV}$ for $\mathrm{KZ}$ (see Materials and Methods part, 2.2.2 Methods subsection). Based on this information one may surmise that the flocculation of suspended particles by CS results from mechanisms which, mainly, imply electrostatic attraction between oppositely charged groups on the particles and chitosan chains (neutralization and patch). In the neutralization mechanism, the surface charges are neutralized by the oppositely charged groups of the polyelectrolytes and the particles attract each other by van der Waals forces, while in the charge patch one, aggregation takes place as a result of the electrostatic attraction between oppositely charged regions on particles [15]. Zeta potential measurements can discriminate between these mechanisms. In case of the charge neutralization mechanism, the maximum removal efficiency takes place at a polymer dose around that required to obtain a zeta potential close to zero [16], while for the patch mechanism, zeta potential needs not to be zero [17]. Zeta potential measurements were undertaken on the same supernatant samples as that used for the absorbance measurements (figure 5, b). Irrespective of the insecticide used, $\zeta$ values increased over the entire domain of chitosan doses investigated, from the initial emulsion negative value (see above) to positive ones, namely $10.2 \mathrm{mV}(F), 14.1 \mathrm{mV}(D c)$ and $18.2 \mathrm{mV}(K Z)$. Worthy of note are the values of $\zeta$, close to zero, recorded in cases of $F$ and $D c$ (between $-4.7 \mathrm{mV}$ and $3.6 \mathrm{mV}$ for $F$ and between $-4.93 \mathrm{mV}$ and $2.33 \mathrm{mV}$ for $D c$ that points to charge neutralization mechanism for both insecticide particles removal by chitosan. The negative value of $\zeta$ at optimum dose $(-11.3 \mathrm{mV})$ noticed for $K Z$ reveals the weaker interactions with chitosan than $F$ and $D c$. This finding could be caused by the more bulky trifluoropropenyl groups in lambdaCyhalothrin than dichlorovinyl and dibromoethenyl in $\alpha$-Cypermethrin and Deltamethrin, respectively, which might impede in a certain extent the polymer-insecticide particles interactions. Also, the slightly higher optimum chitosan dose recorded in case of $F$ than $D c$ and $K Z$ could be assigned to the higher content of active ingredient in $F$ ( $\alpha$-Cypermethrin: $20 \mathrm{mg} \cdot \mathrm{l}^{-1}$ ) than that in $D c$ and $K Z$ (Deltamethrin and lambda-Cyhalothrin: $10 \mathrm{mg} \cdot \mathrm{l}^{-1}$ ).

Influence of insecticide concentration

The insecticides concentration in wastewaters can vary. Therefore, it is necessary to examine the impact of this parameter on the chitosan efficiency in flocculation process. Thus, in addition to the model wastewaters with $c_{\text {ie }}(\%, \mathrm{v} / \mathrm{v})=0.02$, we carried out experiments with insecticide emulsions having $c_{\text {ie }}(\%, \mathrm{v} / \mathrm{v})=0.04$ and 0.06 at the natural emulsions $\mathrm{pH}$.

Figure 4 displays the results obtained in case of $K Z$ which has been chosen as example.

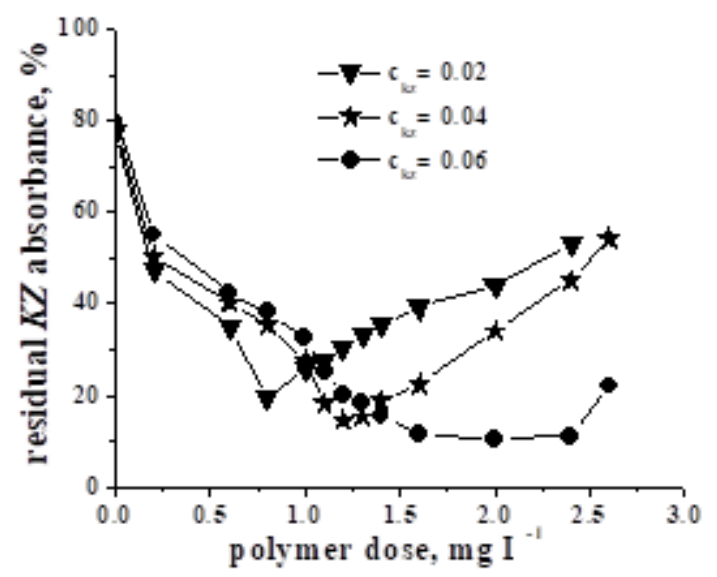

Figure 4. The residual $K Z$ absorbance (\%) dependence on the chitosan dose for different concentrations of $K Z\left(c_{\mathrm{KZ}}\right)$ 
One observes a clear increase of the removal percent of $K Z$ with the increase of $\left(c_{\mathrm{KZ}}\right)$, namely from around $80 \%$ for the lowest $c_{\mathrm{KZ}}$ to around $90 \%$ for the highest one.

Worthy to note is the increase of dose $e_{\mathrm{op}}$ with $c_{\mathrm{KZ}}(\%, \mathrm{v} / \mathrm{v})$, as follows: $0.8 \mathrm{mg} \cdot \mathrm{l}^{-1}$ for $c_{\mathrm{KZ}}=0.02,1.2 \mathrm{mg} \cdot \mathrm{l}^{-1}$ for $c_{\mathrm{KZ}}=0.04,2 \mathrm{mg} \cdot \mathrm{l}^{-1}$ for $c_{\mathrm{KZ}}=0.06$. At higher $\mathrm{KZ}$ concentration, more polycation chains were necessary for the neutralization of particle charge surface, hence the increasing dose op. $_{\text {. }}$

\section{CONCLUSIONS}

1. Wolf equation is proper to describe the viscosity behaviour of chitosan aqueous solution in the presence/absence of salt;

2. The salt addition diminishes the electrostatic repulsive interactions between the charged groups along the chains, and so, the macromolecules adopt a more coiled conformation and hence, the intrinsic viscosity decrease;

3. The increase of flocculants dosage led to the drastic decrease of residual insecticides absorbance until the optimum dose is reached;

4. The optimum chitosan doses (dose $e_{o p} \mathrm{mg} \cdot \mathrm{l}^{-1}$ ) were found to increase as the $K Z$ concentrations increase;

5. Zeta potential data indicate charge neutralization as the common mechanism contributing to the separation of investigated insecticide particles.

\section{ACKNOWLEDGMENT}

This work was supported by a grant of the Romanian National Authority for Scientific Research, CNCS-UEFISCDI, project number PN-III-P4-ID-PCE-2020-0296.

\section{REFERENCES}

[1] Krishnapriya, K.R., Kandaswamy, M., A new chitosan biopolymer derivative as metal-complexing agent: Synthesis, characterization, and metal (II) ion adsorption studies, In: Carbohydr. Res., 2010, 345, 14, 2013-2022, https://doi.org/10.1016/j.carres.2010.06.005

[2] Prado, H.J., Matulewicz, M.C., Cationizaton of polysaccharides: A path to greener derivatives with many industrial applications, In: Eur. Polym. J., 2014, 52, 53-75, https://doi.org/10.1016/j.eurpolymj.2013.12.011

[3] Thomas, S., Ninan, N., Mohan, S., Francis, E., Natural Polymers, Biopolymers, Biomaterials, and Their Composites, Blends, and IPNs, Apple Academic Press Toronto New Jersey, 2013

[4] Dutta, K., Duta, J., Tripathi, V.S., Chitin and Chitosan: chemistry, properties and applications, In: J. Sci. Ind. Res., 2004, 63, 1, 20-31, https://dx.doi.org/10.1016/j.foodhyd.2010.08.008

[5] Costa, C.N., Teixeira, V.G., Delpech, M.C., Souza, J.V.S., Costa, M.A.S., Viscometric study of chitosan solutions in acetic acid/sodium acetate and acetic acid/sodium chloride, In: Carbohydr. Polym., 2015, 133, 245-250, https://doi.org/10.1016/j.carbpol.2015.06.094

[6] Morariu, S., Brunchi, C.-E., Bercea, M., The behavior of chitosan in solvents with different ionic strengths, In: Ind. Eng. Chem. Res. 2012, 51, 12959-12966, https://doi.org/10.1021/ie301309g

[7] Wolf, B.A., Polyelectrolytes revised: Reliable determination of intrinsic viscosities, In: Macromol. Rapid Commun., 2007, 28, 164-170, https://doi.org/10.1002/marc.200600650

[8] Guibal, E., van Vooren, M., Dempsey, B.A., Roussy, J., A review of the use of chitosan for the removal of particulate and dissolved contaminants, In: Sep. Sci. Technol., 2006, 41, 11, 2487-2514, https://doi.org/10.1080/01496390600742807

[9] Ghimici, L., Nichifor, M., Ionic dextran derivatives for removal of Fastac 10 EC from its aqueous emulsions, In: Carbohydr. Polym., 2015, 134, 46-51, https://doi.org/10.1016/j.carbpol.2015.07.100

[10] Ghimici, L., Constantin, M., The separation of the pyrethroid insecticide Fastac 10 EC by cationic pullulan derivatives, In: React. Funct. Polym., 2015, 95, 12-18, https://doi.org/10.1016/j.reactfunctpolym.2015.08.001 
[11] Gamzazade, A.I., Šlimak, V.M., Skljar, A.M., Štykova, E.V., Pavlova, S.-S.A., Rogožin S.V., Investigation of the hydrodynamic properties of chitosan solutions, In: Acta Polymerica, 1985, 36, 8, 420-424, https://doi.org/10.1002/actp.1985.010360805

[12] Brugnerotto, J., Lizardi, J., Goycoolea, F.M., Argüelles-Monal, W., Desbrières, J., Rinaudo, M., An infrared investigation in relation with chitin and chitosan characterization, In: Polymer, 2001, 42, 3569-3580, https://doi.org/10.1016/S0032-3861(00)00713-8

[13] Brunchi, C.-E., Morariu, S., Bercea, M., Impact of ethanol addition on the behaviour of xanthan gum in aqueous media, In: Food. Hydrocolloid, 2021, 120, 106928, https://doi.org/10.1016/j.foodhyd.2021.106928

[14] Sorlier, P., Denuziere, A., Viton, C., Domard, A., Relation between the degree of acetylation and the electrostatic properties of chitin and chitosan, In: Biomacromolecules, 2001, 2, 765-772, https://doi.org/10.1021/bm015531+

[15] Bolto, B., Gregory, J., Organic polyelectrolytes in water treatment, In: Water Res., 2007, 41, 2301-2324, https://doi.org/10.1016/j.watres.2007.03.012

[16] Kleimann, J., Gehin-Delval, C., Auweter, H., Borkovec, M., Super-stoichiometric charge-neutralization in particle-polyelectrolyte systems, In: Langmuir, 2005, 21, 3688-3698, https://doi.org/10.1021/la046911u

[17] Bobacka, V., Eklund, D., The influence of charge density of cationic starch on dissolved and colloidal material from peroxide bleached thermomechanical pulp, In: Colloids Surf. A, 1999, 152, 285-291, https://doi.org/10.1016/S0927-7757(98)00731-6 